\title{
Enfermedad de Kikuchi-Fujimoto: estudio de cuatro casos
}

\author{
M. MONTEJO, N. LEKERIKA, I. ASTIGARRAGA', K. AGUIRREBENGOA, \\ J. GOIKOETXEA, J. G. ERDOZAIN, N. BIDAURRAZAGA² \\ Unidad de Enfermedades Infecciosas. Servicios de ${ }^{1}$ Pediatría y ${ }^{2}$ Anatomía Patológica. \\ Hospital de Cruces. Universidad del País Vasco. Bilbao
}

KIKUCHI-FUJIMOTO DISEASE: REVIEW OF FOUR CASES

\begin{abstract}
RESUMEN
La enfermedad de Kikuchi Fujimoto es una forma inusual de linfadenitis observada inicialmente en Japón. La etiología es desconocida y afecta preferentemente a mujeres jóvenes en forma de linfadenitis cervical dolorosa asociado con frecuencia fiebre y leucopenia. Se trata de una enfermedad benigna y de resolución espontánea. La lesión histológica se caracteriza por fenómenos necróticos con cariorrexis, pérdida parcial de la estructura ganglionar y focos de histiocitos en la zona cortical y/o paracortical. Un hallazgo característico es la ausencia de granulocitos neutrófilos, lo que la diferencia de otras linfadenitis necrotizantes.

Recogemos cuatro casos de enfermedad de Kikuchi-Fujimoto atendidos en nuestro centro en un año.

Tres pacientes eran adultos y el otro una niña en edad pediátrica. La mitad referían fiebre y los cuatro tuvieron adenopatías. La evolución fue buena en todos.

Pensamos que la enfermedad de Kikuchi-Fujimoto es probablemente más frecuente en nuestro país que lo recogido en la literatura.
\end{abstract}

PALABRAS CLAVE: Enfermedad de Kikuchi-Fujimoto. Linfadenitis.

\begin{abstract}
Kikuchi Fujimoto disease is an uncommon form of lymphadenitis, firstly described in Japan. Etiology is unknown. It affects mainly young women. It commonly manifests as a painful cervical lymphadenitis usually associated with fever and leukopenia. Clinical course users to be benign, leading spontaneously to a complet recovery. Histological findings include necrotizing changes with cariorrhesis, partial loss of ganglionar architecture and foci of histiocytic infiltrates in the cortical and/or paracortical zones of the lymph nodes. A common findings is the absence of neutrophil granulocytes in the inflammatory infiltrates, in contrast to other necrotizing lymphadenitis.

We report four cases of Kikuchi Fujimoto disease, recently identified in our hospital.
\end{abstract}

KEY WORDS: Kikuchi-Fujimoto disease. Lymphadenitis.

Montejo M, Lekerika N, Astigarraga I, Aguirrebengoa K, Goikoetxea J, Erdozain JG, Bidaurrazaga N. Enfermedad de Kikuchi-Fujimoto: estudio de cuatro casos. An Med Interna (Madrid) 2006; 23: 173-175.

\section{INTRODUCCIÓN}

La enfermedad de Kikuchi Fujimoto o linfadenitis necrotizante no granulocítica fue descrita por primera vez en la literatura japonesa en 1972 (1-4). Aunque la mayor parte de los casos inicialmente descritos pertenecen a países orientales, la distribución geográfica de la enfermedad parece ser generalizada. No es rara en América o Europa, donde los primeros casos se describen a partir de 1982, y concretamente en nuestro país, en el año 1985 (5).

$\mathrm{Su}$ etiología es desconocida y se han involucrado en la misma tanto factores infecciosos como autoinmunes. Algunos autores consideran que la enfermedad sería el resultado de una reacción inmunológica del organismo inducida por infecciones virales, preferentemente: virus de Epstein Barr (VEB), Herpesvirus humano tipo 6, Parvovirus B19 y Citomegalovirus (CMV).

Recogemos cuatro casos de enfermedad de Kikuchi Fujimoto atendidos en el último año en nuestro hospital y cuyo motivo de ingreso fue la presencia de fiebre y/o adenopatías.

\section{CASOS APORTADOS}

Caso 1. Varón de 20 años de edad, sin antecedentes de interés. Ingresó por presentar desde hacía un mes adenopatías laterocervica- 
les izquierdas y odinofagia. Los últimos 15 días se asoció fiebre, astenia, mialgias y pérdida de $5 \mathrm{~kg}$ de peso. La exploración fue normal salvo la presencia de fiebre $\left(38^{\circ} \mathrm{C}\right)$, conglomerado adenopático látero-cervical izquierdo y mínimas adenopatías latero-cervicales derechas. Se le practicó un TAC cervical que mostraba adenopatías cervicales, analítica de sangre con leucopenia y trombopenia y biopsia ganglionar informada de linfadenitis aguda. En la analítica destacaba: leucocitos 2.500 con $26 \%$ linfocitos, plaquetas 57.000 y LDH $816 \mathrm{U} / \mathrm{L}$.

La PPD 5U fue negativa y los hemocultivos, coprocultivo RPR y serologías frente a CMV, VEB, Brucella, Leptospira, Francisella tularensis y Bartonella fueron así mismo negativas. Ante la presencia de la fiebre se practicó nueva biopsia cervical observándose a nivel cortical-paracortical una linfadenitis necrotizante parcheada, con abundantes restos celulares y rodeada por linfocitos $\mathrm{T}$ CD4+, histiocitos CD68, escasos polimorfonucleares y células plasmáticas.; características histopatológicas que definen la enfermedad de Kikuchi. Se inició tratamiento con antiinflamatorios no esteroides con desaparición de la fiebre. Año y medio después sigue asintomático.

Caso 2. Varón de 30 años de edad sin antecedentes previos, que ingresó por presentar fiebre y astenia desde hacía 20 días. En la exploración además de fiebre $\left(39^{\circ} \mathrm{C}\right)$, presentaba una adenopatía supraclavicular derecha de $1 \mathrm{~cm}$ de diámetro y otras tres algo más pequeñas en región latero-cervical derecha y adenopatías menores de $1 \mathrm{~cm}$ en región latero-cervical izquierda. En la analítica los únicos datos reseñables eran una tasa de leucocitos de 3.500 con $40 \%$ linfocitos y LDH de $626 \mathrm{U} / \mathrm{L}$. La PPD 5U fue negativa y los hemocultivos, coprocultivo y pruebas serológicas frente a $\mathrm{Bru}$ cella, Leptospira, Francisella tularensis, Bartonella, VEB, y CMV fueron negativas. La TAC cérvico-torácico puso de manifiesto la presencia de adenopatías cervicales bilaterales de hasta 13 $\mathrm{mm}$ de diámetro a nivel de cadena yugulo-digástrica derecha y en los espacios cervicales posteriores de carácter inespecífico y la existencia de adenopatías axilares bilaterales de hasta $1 \mathrm{~cm}$ en axila derecha. Practicada la biopsia de una adenopatía cervical se llegó al diagnóstico de linfadenitis necrotizante paracortical, con abundante cariorrexis, y células inflamatorias mononucleares. Se inició tratamiento con antiinflamatorios no esteroideos desapareciendo la fiebre y encontrándose asintomático. En la actualidad lleva diez meses sin recidivar.

Caso 3. Niña de 8 años de edad remitida por su pediatra al hospital para estudio de adenopatía cervical dolorosa de una semana de evolución, sin respuesta al tratamiento con amoxicilina-clavulánico. No refería fiebre, astenia, anorexia ni otra clínica asociada. A la exploración se apreciaron adenopatías laterocervicales izquierdas menores de $1 \mathrm{~cm}$ y adenopatía laterocervical inferior de $3,5 \times 2 \mathrm{~cm}$ de consistencia dura, no adherida, sin signos inflamatorios asociados. En las pruebas complementarias destacaba hemograma, bioquímica y coagulación normales, VSG $30 \mathrm{~mm}$, anticuerpos antinucleares y factor reumatoideo negativos. PPD 5U negativo. Serología Herpesvirus 6 IgM positiva. Serologías CMV, VEB, VHS I - II, Hepatitis B y C, VIH, Toxoplasma gondii, Bartonella henselae, Parvovirus B19 y Salmonella typhi negativas. La Rx de tórax y la ecografía abdominal fueron normales y en la ecografía cervical se confirmaron las adenopatías.

Ante la persistencia de las adenopatías se practicó biopsia abierta a los 18 días de evolución. El estudio histológico mostró focos de necrosis paracortical, rodeados por linfocitos T CD4 y CD8 + y abundantes histiocitos CD68+, entremezclados con células plasmocitoides y ausencia significativa de neutrófilos. La tinción de Auramina, cultivo para micobacterias, PCR de Enterovirus, HSV, VVZ, CMV, VEB y Herpesvirus tipos 6, 7 y 8 del tejido ganglionar fueron negativos. El curso de la enfermedad fue favorable y autolimitado. Dos años después se encuentra asintomática.

Caso 4. Mujer de 25 años, que ingresó por adenopatías en cadena yugular izquierda. Destacaba como único antecedente epidemiológico picaduras de numerosos insectos en un viaje a Méjico realizado hacía un mes. En el examen físico se palpaban varias adenopatías de alrededor de $1 \mathrm{~cm}$ de diámetro en zona laterocervical izquierda, no dolorosas, siendo el resto de la exploración normal. En la analítica solamente destacaba leucopenia (4.300 leucocitos/ul) con fórmula normal y LDH 372. ANAS negativos. Serología frente a Bartonella henselae, Salmonella, Brucella, CMV, EBV, virus hepatitis B, C, rubéola y RPR: negativas. Practicado TAC torácico-abdominal fue normal. Realizada biopsia de adenopatía laterocervical izquierda mostró focos de necrosis paracortical con componente inflamatorio polimorfo, constituido por linfocitos T CD4+, histiocitos CD 68, escasas células plasmáticas y ausencia de eosinófilos. La paciente evolucionó favorablemente sin tratamiento alguno y permanece asintomática dos años después.

\section{DISCUSIÓN}

La enfermedad de Kikuchi-Fujimoto es una forma benigna y autolimitada de linfadenitis necrotizante, con mayor prevalencia en mujeres jóvenes, caracterizada clínicamente por la existencia de linfadenopatías, fundamentalmente laterocervicales $(6,7)$ y supraclaviculares, junto con un síndrome febril prolongado (8). La exploración física y analítica suelen ser normales aunque puede encontrarse leve trombocitopenia, neutropenia, alteraciones de las enzimas hepáticas y un aumento de la LDH. Raramente se ha descrito afectación extranodal en riñón, hígado, tracto gastrointestinal, glándulas suprarrenales, glándulas paratiroideas, médula ósea y SNC $(2,9)$.

El diagnóstico definitivo se basa en el examen histológico en el que encontramos una hiperplasia reactiva con focos bien circunscritos de necrosis paracortical con abundantes detritus celulares, rodeados por un infiltrado inflamatorio de linfocitos CD4+, histiocitos CD68, y quizás algunos inmunoblastos; con escasa presencia de polimorfonucleares neutrófilos y células plasmáticas (13-15).

Se describen tres subtipos histológicos: necrotizante, proliferativo y xantomatoso, que en realidad no son más que distintos periodos en diferentes momentos evolutivos de la enfermedad $(10,11)$.

En el diagnóstico diferencial se deben incluir los linfomas, las linfadenitis bacterianas, toxoplasmosis, el lupus eritematoso diseminado (5).

Por lo general, la enfermedad se resuelve por sí sola en varias semanas o meses. Aproximadamente el 3-4\% de los enfermos presentan recurrencia de la enfermedad, a veces hasta varios años después del episodio inicial. No existe tratamiento específico y sólo en los casos muy sintomáticos los pacientes pueden beneficiarse de la administración de antiinflamatorios no esteroideos o esteroides a bajas dosis (12). 


\section{Bibliografía}

1. Hudnall D. Kikuchi-Fujimoto Disease. Is Epstein-Barr Virus the Culprit? Am J Clin Pathol 2000; 113: 761-764.

2. Calvo Romero JM. Kikuchi-Fujimoto disease (histiocytic necrotizing lymphadenitis). Rev Clin Esp 2002; 202: 94-95.

3. Norris AH. Kransinskas AM, Salhany KE, Gluckman SJ. Kikuchi-Fujimoto disease: a benign cause of fever and lymphadenopathy. Am J Med 1996; 101: 401-5.

4. Payne JH, Evans M, Gerrard MP. Kikuchi-Fujimoto disease: a rare but important cause of lymphadenopathy. Acta Pediatr 2003; 92: 261-264.

5. Veiga-González M, Fresno-Forcelledo MF, Pérez del Río MJ, TriviñoLópez A, Riera-Velasco JR, Herrero-Zapatero A. Linfadenitis necrotizante no granulocítica (enfermedad de Kikuchi). Análisis morfológico de tres casos con especial atención al diagnóstico diferencial. Patología 1996; 29: 295-302.

6. Lozano Parras MA, Anguita Alonso P, Cigüenza Gabriel R, Calvo Manuel E, Alba Losada J, Espinós Pérez D. Enfermedad de Kikuchi: a propósito de un caso y revisión de la literatura. An Med Interna (Madrid) 2003; 20: 247-250.

7. Gómez García AM, Martínez Hurtado E, Ruiz Ribera I. Enfermedad de Kikuchi-Fujimoto asociada a infección por virus de parotiditis . A propósito de 1 caso. An Med Interna (Madrid) 2004; 21: 135-137.

8. Blewitt RW, Kumar SN, Abraham JS. Recurrence of Kikuchi's lymphadenitis after 12 years. J Clin Pathol 2000; 53: 157-158.
9. Tsai MK, Huang HF, Hu RH, Lee PH, Lee CJ, Chao SH, et al. Fatal Kikuchi-Fujimoto disease in trasplant recipients: a case report. Trasplant Proc 1998; 30: 3137-8.

10. Menasce LP, Banerjee SS, Edmondson D, Harris M. Histiocytic necrotizing lymphadenitis (Kikuchi-Fujimoto disease): continuing diagnostic difficulties. Histopathology 1998; 33: 248-254.

11. Chiu CF, Chow RC, Lin TY, Tsai MH, Shih CM, Chen LM. Virus Infection in Patients With Histiocytic Necrotizing Limphadenitis in Taiwan. Detection of Epstein-Barr Virus, Type I Human T-Cell Lymphotropic Virus, and Parvovirus B19. Am J Clin Pathol 2000; 113: 774-781.

12. Lorenzo Hernández A. Gamallo Amat C, Ortega Martínez de la Victoria L, Cabestrero Alonso D, Camacho Siles J, Arnalich Hernández F, et al. Linfadenitis necrotizante histiocitaria: enfermedad de Kikuchi-Fujimoto. A propósito de 6 casos. An Med Interna (Madrid) 1999; 16: 301-4.

13. Yoshino T, Mannami T, Ichimura K, Takenata K, Nose S, Yamadori I Akagi T. Two cases of histiocytic necrotizing lymphadenitis following diffuse large B-cell lymphoma. Hum Pathol 2000; 31: 1328-1331.

14. Takakuwa T, Ohnuma S, Koike J, Hoshikawa M, Koizumi H. Involvement of cell-mediated killing in apoptosis in histiocytic necrotizing lymphadenitis. Histopathology 1996; 28: 41-48.

15. Lin HC. Su CY, Huang CC, Hwang CF, Chien CY. Kikuchi's disease: a review and analysis of 61 cases. Otolaryngol Head Neck Surg 2003; 128: $650-653$ 